\title{
Short Term Evaluation of the Effects of Orthodontic Clear Aligners on Sleep Bruxism Activity
}

\author{
Andrea Bargellini, ${ }^{1,2,}$ Tommaso Castroflorio, ${ }^{1,2}$ Federica Casasco, ${ }^{1,2}$ Maria Giacone, ${ }^{1,2}$ Francesco \\ Garino, ${ }^{3}$ Giovanni Cugliari, ${ }^{4,5}$ and Andrea Deregibus ${ }^{1,2}$ \\ ${ }^{1}$ Department of Surgical Sciences, Specialization School of Orthodontics, Dental School, University of Torino, Via Nizza 230,10126 Torino, Italy \\ ${ }^{2}$ Department of Surgical Sciences, Gnathology Unit, Dental School, University of Torino, Via Nizza 230,10126 Torino, Italy \\ ${ }^{3}$ Private Practice, Corso Matteotti 0, 10100 Torino, Italy \\ ${ }^{4}$ Department of Statistics and Quantitative Methods, University of Milano-Bicocca, Via Bicocca Degli Arcimboldi 8, 20126, Milan, Italy \\ ${ }^{5}$ Department of Medical Sciences, University of Torino, C.So Dogliotti 14,10126 Torino, Italy \\ Corresponding author: Andrea Bargellini, Department of Surgical Sciences, Specialization School of Orthodontics, Dental School, University of Torino, Via Nizza 230,10126 \\ Torino, Italy. Tel: +39-3339233219, E-mail: bargelli@ipsnet.it
}

Received 2016 July 18; Accepted 2016 September 18.

\begin{abstract}
Background: In the last years an increasing number of patients have sought for comfortable and aesthetic alternatives to fixed appliances. Aligners, due to the protection against tooth wear, can be compared to occlusal splints used in patients suffering from sleep bruxism (SB). The aim of the study was to analyze the effects of clear aligners on SB episodes using a validated portable device (Bruxoff®, OTBioelettronica, Torino, Italy) allowing a simultaneous recording of electromyographic signals from both the masseter muscles as well as heart frequency to evaluate variation on SB activity.

Methods: Forty patients were analyzed for the study. Twenty of them were assigned to orthodontic treatment with clear aligners (Invisalign $®$, Align Technology). The other twenty patients were used as control group observed for a period of twelve months. Intra-group and inter-group variances were analyzed by using a two-way ANOVA test. For each analysis a $\mathrm{P}<0.05$ was set.

Results: After the first month of clear aligners therapy, all patients in the case group showed a significant reduction in the number of SB episodes $(\mathrm{P}<0.05)$. However, after three months SB values returned to baseline levels. The number of tonic contractions were reduced even after three months of orthodontic treatment $(\mathrm{P}<0.05)$.

Conclusions: While clear aligners seem to be capable to reduce clenching, i.e. occlusal load, in SB patients, the grinding activity seems to be not influenced by those appliances at least in the short term of the present investigation.
\end{abstract}

Keywords: Sleep Bruxism, Clear Aligners, Orthodontics, Surface Electromyography, Masseter Muscle, Heart Rate

\section{Background}

Sleep bruxism (SB) is a sleep-related motor disorder characterized by (rhythmic) involuntary phasic or tonic (sustained) motor activity in the masticatory muscles (e.g. masseter, temporalis) during sleep (1). SB shows a mean prevalence of $14 \%-20 \%$ in adolescents and $12.8 \%$ in adults, with no gender differences and a tendency to decline with increasing age $(1,2)$. Most of the orthodontic patients are part of this population $(3,4)$. In a recent systematic literature review4 dental malocclusion presents moderate association with SB in adolescents and, moreover, the presence of parafunctions such as bruxism may be a predisposing factor for temporo-mandibular disorders (TMDs) (5). Since the association between SB and TMDs with painful sensation has been stated (6-8) and occlusal overload induced by SB may be a co-factor (9-12), the orthodontic treatment of malocclusion in patients suffering from SB may be useful in controlling, at least, one of the factors involved in the multifactorial etiology of TMDs. In the last years, the use of clear aligners in orthodontic treatment has reached an important role even in complex cases $(13,14)$. Such disposals, due to the protection against tooth wear, can be compared to occlusal splints used in patients suffering from SB(15-19). Unfortunately, no literature data are available about the efficacy and efficiency of these orthodontic disposals in modifying or reducing the number of SB episodes. The study was designed to answer the clinical research question "is sleep bruxism influenced by orthodontic treatment with clear aligners?"

\section{Objectives}

The source underlying the study was that a positive answer to the above question could lead to the possible introduction of a specific orthodontic treatment for patients suffering from SB with a possible modulation of the number of SB episodes per night. 


\section{Methods}

This observational prospective study, involved 53 subjects ( $27 \mathrm{~m}, 25 \mathrm{f}, 28 \pm 13$ years) selected among patients referring to the Orthodontics Unit of the Dental School of the University of Torino in the period February-December 2015 requiring an orthodontic treatment. All the subjects were screened for sleep bruxism with a validated portable device (Bruxoff @, OT Bioelettronica, Torino, Italy) (20-24). On the basis of this evaluation the subjects were divided into two groups: the case group composed by 20 patients $(7 \mathrm{~m}$, $13 \mathrm{f}, 25 \pm 10$ years), and the control group composed by 33 patients ( $20 \mathrm{~m}, 13 \mathrm{f}, 26 \pm 10$ years). The control group was a "waiting list" control group, without any form of treatment for the following 6 months. During the follow-up period, 13 patients from the control group ( $5 \mathrm{~m}, 8 \mathrm{f}, 28 \pm$ 7 years) withdraw from the study, obtaining a final control group formed by 20 patients ( $15 \mathrm{~m}, 5 \mathrm{f}, 29 \pm 12$ years). The study was conducted according to the Declaration of Helsinky, and each subject was aware to withdraw from the experiment at any time. A written informed consent was obtained by each patient. The study was approved by the Local ethical committee (\#3742015).

\subsection{Inclusion and Exclusion Criteria}

The inclusion and exclusion criteria are resumed in Table 1.

Table 1. Inclusion and Exclusion Criteria

\begin{tabular}{l|l}
\hline Inclusion Criteria & Exclusion Criteria \\
\hline Patients aged from 13 to 30 years & Previous or current SB treatment \\
\hline $\begin{array}{l}\text { Patients requiring orthodontic } \\
\text { treatment }\end{array}$ & $\begin{array}{l}\text { Presence of prosthodontics } \\
\text { rehabilitations }\end{array}$ \\
\hline \multirow{2}{*}{ Absence of medications } & $\begin{array}{l}\text { Missing teeth, with the exception of } \\
\text { the third molars }\end{array}$ \\
\hline \multirow{4}{*}{$\begin{array}{l}\text { Avoiding alcohol, nicotine or } \\
\text { caffeine during night recordings }\end{array}$} & Periodontal disease \\
\cline { 2 - 2 } & $\begin{array}{l}\text { Group II and/or Group III TMDs } \\
\text { (articular TMDs) }\end{array}$ \\
\cline { 2 - 2 } & $\begin{array}{l}\text { Medical history of neurological, } \\
\text { periodic leg movements, insomnia) }\end{array}$ \\
\cline { 2 - 2 } & Post-traumatic patients \\
\cline { 2 - 2 } & $\begin{array}{l}\text { Previous or current orthodontic } \\
\text { treatment }\end{array}$ \\
\cline { 2 - 2 } & $\begin{array}{l}\text { Obstructive sleep apnea syndrome } \\
\text { (OSAS) }\end{array}$ \\
\hline
\end{tabular}

\subsection{Clinical Records}

Dental panoramics, latero-lateral radiograms and dental casts were taken for all the 20 patients before to start the orthodontic treatment with clear aligners.

\subsection{SB Diagnosis and Monitoring}

A validated portable device for SB diagnosis (Bruxoff $®$, OT Bioelettronica, Torino, Italy) with three channels was used for recording (20-24). Two channels were used to acquire surface electromyography (sEMG) from the masseter (bilaterally), and the third channel was used to acquire the heart frequency (HF). The three signals were sampled at $800 \mathrm{~Hz}$, with 8 bit resolution.

A microSD card was used to store the data as a binary file. The sEMG channels were filtered between 10 and $400 \mathrm{~Hz}$ with gain 4300. The electrocardiographic (ECG) channel was filtered between 15 and $160 \mathrm{~Hz}$ with gain 700. Surface EMGs from the masseter muscles (both sides) were detected with disposable bipolar concentric electrodes (Code $®$, Spes Medica, Battipaglia, Italy), with a radius of $16 \mathrm{~mm}$ and with detection site made of $\mathrm{AgCl}$ (Figure 1). The concentric-ring systems of the Code electrodes show higher spatial selectivity with respect to the traditional detection systems and reduce the problem of electrode location since they are invariant to rotations and reducing EMG crosstalk (25). The heart frequency was perceived with a disposable bipolar electrode located on the left side of the thorax just below the pectoral muscles. At first, the subjects were asked to accomplish three maximum voluntary clenching (MVC), 3 seconds each and separated by 10 seconds of rest. The greatest of the MVC measures was used to normalize the EMG values as a percent of MVC. Previous studies showed a high sensitivity (92.3\%) and specificity (91.6\%) of the portable device when the cut-off was set at 4 SB episodes per hour accordingly to published criteria (22). Furthermore a reliability study of the Bruxoff demonstrated a good reproducibility for SB episodes per night, SB episodes per hour, and heart frequency20. Scoring on the Bruxoff recordings was automatically performed by dedicated software (Bruxmeter $₫$, OT Biolettonica, Torino, Italy).

The SB episode can be classified by the software, if the sEMG burst is greater than 10\% MVC and if it immediately follows ( 1 - 5 seconds interval) a heart rate increase of $20 \%$ with respect to the baseline accordingly to the existing literature (25-27). Moreover, instrumental evaluation allowed to identify specific sEMG signals: tonic, phasic and mixed.

\subsection{Night Recordings With Bruxoff Device}

Multiple night recordings with the Bruxoff® device were made both for case and control groups. The first night was used as accommodation session. Then other recordings were made at screening time, at the beginning of orthodontic treatment (only for case group) (T0), after 1 month (T1) and after three months (T2). The device 
Figure 1. The Bruxoff $®$ and the CoDe® (Spes Medica, Genova, Italy) Electrode Used in This Study for the Detection of Myoeletric Signals From the Masseter Muscles

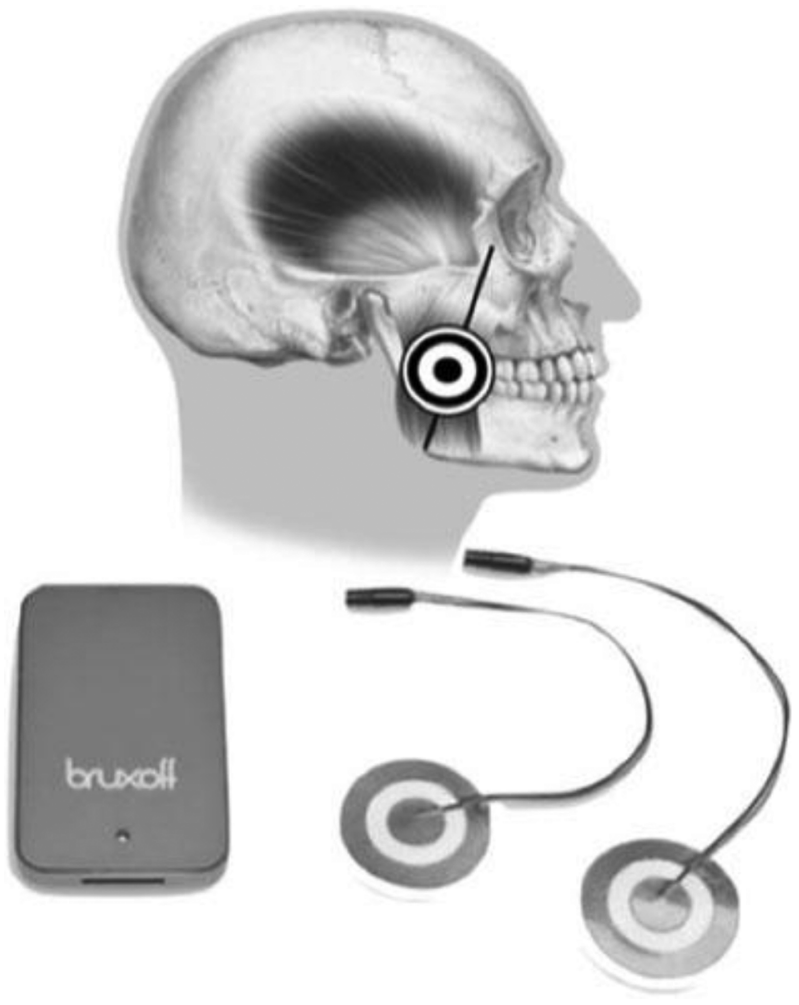

The concentric-ring systems of the Code electrodes show higher spatial selectivity with respect to the traditional detection systems and reduce the problem of electrode location since they are invariant to rotations and reducing EMG crosstalk. At the top a schematic representation of the electrode location over the masseter muscle is shown. Black line: gonial angle-cantus line used as anatomical landmark.

was used and mounted without technical assistance, after prior training, at home. Written instructions were provided and a night-time telephone number to call in the event of difficulties.

\subsection{Orthodontic Treatment}

Orthodontic diagnosis was completed by the digital scanning of dental impression through dedicated software (Clincheck $®$ Align Technology Inc. San Jose, California (USA)). This software allowed virtual simulations of orthodontic movements and guided the realization of custom-made clear aligners for each patient. All patients were provided with a precise sequence of clear aligners, to be replaced every two weeks, according to the standard protocol (28). Every month each patient was controlled in order to check compliance, orthodontic tooth movement and to perform routine inspections.

\subsection{Statistical Analysis}

The number of SB episodes of every patient, recorded during the 3 time points (To, T1, T2), was collected. The sample passed the Kolmogorov-Smirnov normality test. Intragroup and inter-group variances were analyzed with twoway (variables time and treatment) ANOVA test. All statistical procedures were performed with the software statistical package for the social science v. 23.0 (SPSS 23.0®, IBM, Milan, Italy). Significance was set at $\mathrm{P}<0.05$.

\section{Results}

The two groups of patients were followed for a period of three months and evaluated in three different moments (T0: screening, T1: evaluation after 1 month, T2: evaluation after three months) with instrumental (Bruxoff $®$ ) recordings. The statistical analysis demonstrated that the number of SB episodes significantly reduced after one month follow-up in the case group $(\mathrm{P}<0.05,95 \% \mathrm{CI})$ (Figure 2). However, at T2 evaluation the number of SB episodes in the case group raised up to To levels with no statistical differences between T0 and T2 (P>0.05, 95\% CI). The number of total tonic contractions of the masseters muscles in the case group reduced in a significant way after one and three months $(\mathrm{P}<0.05,95 \% \mathrm{CI})$ (Figure 3), although the number of tonic contractions related to SB episodes in the case group, after a significant reduction in the first month $(\mathrm{P}<$ $0.05,95 \% \mathrm{CI}$ ), raised up to To levels with no statistical differences between T0 and T2 (P > 0.05, 95\% CI) (Figure 4). Phasic and mixed contractions of the masseters muscles showed no statistical differences at $\mathrm{T} 1$ and $\mathrm{T} 2$ points neither inter-groups nor intra-groups $(\mathrm{P}>0.05,95 \% \mathrm{CI})$.

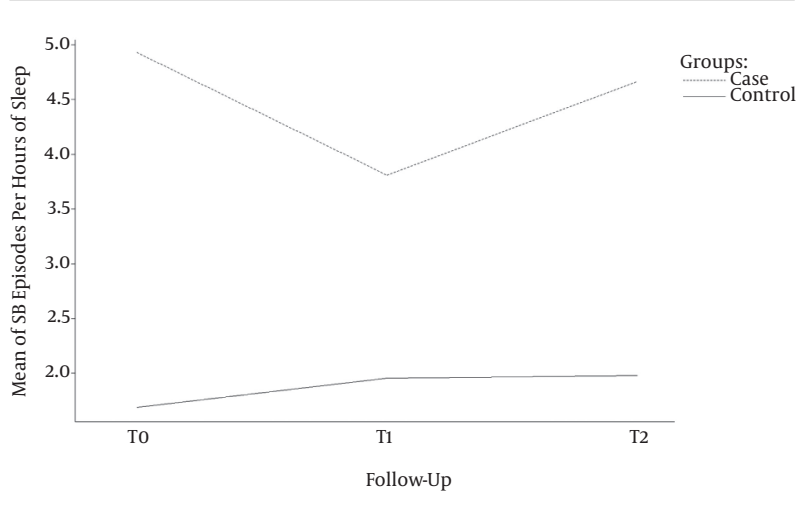

Figure 2. Number of SB Episodes Per Hour of Sleep

\section{Discussion}

In the last years, the use of clear aligners to obtain orthodontic tooth movement grew up significantly in order 


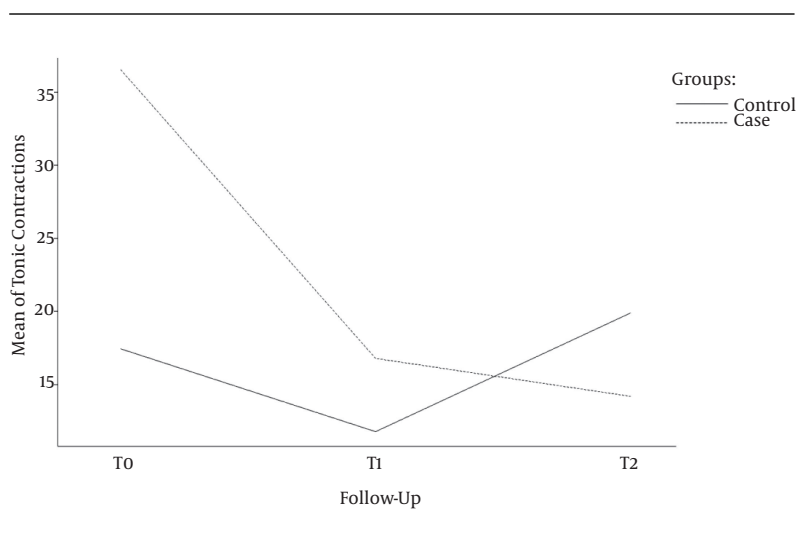

Figure 3. Mean of General Tonic Contractions

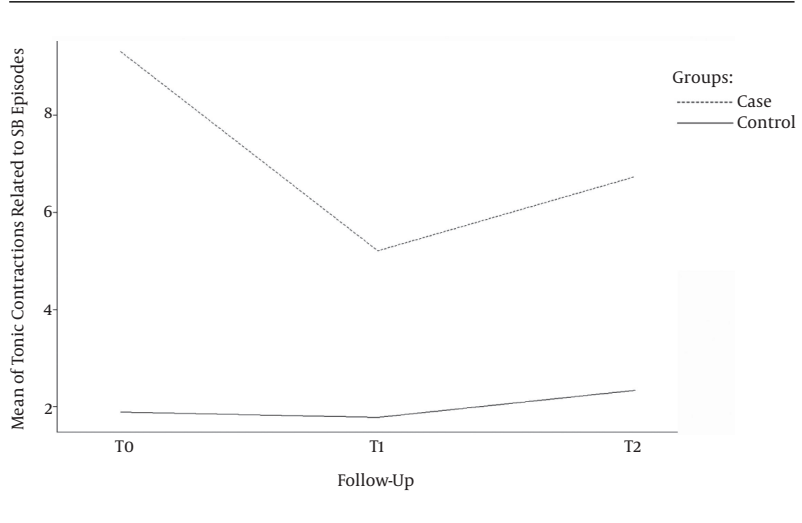

Figure 4. Mean of Tonic Contractions Related to SB Episodes

to answer the request for possible comfortable and aesthetics alternatives to traditional fixed appliances. Such disposals, due to the protection against tooth wear, can be compared to occlusal splints used in patients suffering from SB. Results from the present study showed that, on a short term basis, the use of clear aligners can reduce tonic contractions (i.e. clenching) but not phasic (i.e. grinding contractions). Furthermore the number of SB episodes showed a significant reduction during the first month of treatment, while after three months no significant differences were observed with respect to the baseline. Interestingly $38 \%$ of the patients treated with Invisalign $®$ aligners were low intensity bruxers while the $62 \%$ were high frequency bruxers at To according to Rompré et al. (2007) (29). After three months of treatment the $54 \%$ of patients were low frequency bruxers while the $46 \%$ were high frequency bruxers. Accordingly to the statistical analysis the percentage reduction could be associated to the significant reduction of SB related clenching contractions. According to Dubé et al. (2004) (30), patients wearing oral appliances (OAs) significantly reduced after one month the number of SB episodes per hour of sleep such as for the number of masseters contractions (bursts per hour of sleep) in polysomnographic recordings. According to Manfredini et al. (2015) (15) it seems that almost every type of OA is in some way effective to reduce SB activity. This may suggest the existence of a possible 'novelty effect' associated with the use of an OA, which leads to a reduction in sleep-time masticatory muscles' activity, possibly due to the transient need for reorganizing motor unit employment. All patients recruited for this study were selected according to the presence or absence of SB. All of them did not present clinical signs or symptoms of SB, but after instrumental recording with Bruxoff $®$ device, for the $62 \%$ of them severe SB was diagnosed. Moreover, clinical signs and symptoms of SB cannot be used for definite diagnosis $(2,22$, 23), especially after analyzing preliminary data from this study. Since the association between SB and TMDs with painful sensation has been stated (6-8) and occlusal overload induced by SB may be a co-factor (9-12), aligner treatment should be considered as a possible option to treat patients suffering from SB with need for orthodontic treatment. In this light the use of validated portable devices (22) for the diagnosis of SB should be introduced in the orthodontics clinical setting at least as screening devices.

\subsection{Conclusions}

While clear aligners seem to be capable to reduce clenching, i.e. occlusal load, in SB patients, the grinding activity seems to be not influenced by those appliances at least in the short term of the present investigation.

\section{Acknowledgments}

The procedures were approved by the ethics committee of the Lingotto dental school. All individuals gave their informed consent in accordance with the Helsinki Declaration.

\section{Footnote}

Funding/Support: The research funding was provided by University of Torino.

\section{References}

1. Lavigne GJ, Khoury S, Abe S, Yamaguchi T, Raphael K. Bruxism physiology and pathology: an overview for clinicians. J Oral Rehabil. 2008;35(7):476-94. doi: 10.1111/j.1365-2842.2008.01881.x. [PubMed: 18557915].

2. Manfredini D, Winocur E, Guarda-Nardini L, Paesani D, Lobbezoo F. Epidemiology of bruxism in adults: a systematic review of the literature. J Orofac Pain. 2013;27(2):99-110. doi: 10.11607/jop.921. [PubMed: 23630682]. 
3. Castroflorio T, Bargellini A, Rossini G, Cugliari G, Deregibus A. Sleep bruxism in adolescents: a systematic literature review of related risk factors. EurJOrthod. 2016 doi: 10.1093/ejo/cjw012. [PubMed: 26884421].

4. Castroflorio T, Bargellini A, Rossini G, Cugliari G, Rainoldi A, Deregibus A. Risk factors related to sleep bruxism in children: A systematic literature review. Arch Oral Biol. 2015;60(11):1618-24. doi: 10.1016/j.archoralbio.2015.08.014. [PubMed: 26351743].

5. Ohrbach R, Bair E, Fillingim RB, Gonzalez Y, Gordon SM, Lim PF, et al. Clinical orofacial characteristics associated with risk of firstonset TMD: the OPPERA prospective cohort study. J Pain. 2013;14(12 Suppl):T33-50. doi: 10.1016/j.jpain.2013.07.018. [PubMed: 24275222].

6. Fernandes G, van Selms MK, Goncalves DA, Lobbezoo F, Camparis CM. Factors associated with temporomandibular disorders pain in adolescents. J Oral Rehabil. 2015;42(2):113-9. doi: 10.1111/joor.12238. [PubMed: 25244610].

7. Franco-Micheloni AL, Fernandes G, de Godoi Goncalves DA, Camparis CM. Temporomandibular Disorders in a Young Adolescent Brazilian Population: Epidemiologic Characterization and Associated Factors. J Oral Facial Pain Headache. 2015;29(3):242-9. doi: 10.11607/ofph.1262. [PubMed: 26244432].

8. McNamara JA, Seligman DA, Okeson JP. Occlusion, Orthodontic treatment, and temporomandibular disorders: a review. J Orofac Pain. 1995;9(1):73-90. [PubMed: 7581209].

9. Simmons JH. Neurology of sleep and sleep-related breathing disorders and their relationships to sleep bruxism. J Calif Dent Assoc. 2012;40(2):159-67. [PubMed: 22416635].

10. Dao TT, Lund JP, Lavigne GJ. Comparison of pain and quality of life in bruxers and patients with myofascial pain of the masticatory muscles. J Orofac Pain. 1994;8(4):350-6. [PubMed: 7670422].

11. Arima T, Svensson P, Arendt-Nielsen L. Experimental grinding in healthy subjects: a model for postexercise jaw muscle soreness? JOrofac Pain. 1999;13(2):104-14. [PubMed: 10425982].

12. Lund J. P. , Lavigne G. J. , Dubner R. , Sessle B. J. . From Basic Science to Clinical Management. Quintessence, Sao Paulo; 2002.

13. Hennessy J, Al-Awadhi EA. Clear aligners generations and orthodontic tooth movement. I Orthod. 2016;43(1):68-76. doi: 10.1179/1465313315Y.0000000004. [PubMed: 25939782].

14. Dasy H, Dasy A, Asatrian G, Rozsa N, Lee HF, Kwak JH. Effects of variable attachment shapes and aligner material on aligner retention. Angle Orthod. 2015;85(6):934-40. doi: 10.2319/091014-637.1. [PubMed 26516708].

15. Manfredini D, Ahlberg J, Winocur E, Lobbezoo F. Management of sleep bruxism in adults: a qualitative systematic literature review. J Oral Rehabil. 2015;42(11):862-74. doi: 10.1111/joor.12322. [PubMed: 26095208].

16. Dao TT, Lavigne GJ, Charbonneau A, Feine JS, Lund JP. The efficacy of oral splints in the treatment of myofascial pain of the jaw muscles: a controlled clinical trial. Pain. 1994;56(1):85-94. [PubMed: 8159444].

17. Raphael KG, Marbach JJ, Klausner JJ, Teaford MF, Fischoff DK. Is bruxism severity a predictor of oral splint efficacy in patients with myofascial face pain? J Oral Rehabil. 2003;30(1):17-29. [PubMed: 12485379].

18. Macedo $C R$, Silva $A B$, Machado MA, Saconato $H$, Prado GF.
Occlusal splints for treating sleep bruxism (tooth grinding). Cochrane Database Syst Rev. 2007(4):005514. doi: 10.1002/14651858.CD005514.pub2. [PubMed: 17943862].

19. Klasser GD, Greene CS. Oral appliances in the management of temporomandibular disorders. Oral Surg Oral Med Oral Pathol Oral Radiol Endod. 2009;107(2):212-23. doi: 10.1016/j.tripleo.2008.10.007. [PubMed: 19138639].

20. Castroflorio T, Mesin L, Tartaglia GM, Sforza C, Farina D. Use of electromyographic and electrocardiographic signals to detect sleep bruxism episodes in a natural environment. IEEE J Biomed Health Inform. 2013;17(6):994-1001. doi: 10.1109/JBHI.2013.2274532. [PubMed: 24240717].

21. Deregibus A, Castroflorio T, Bargellini A, Debernardi C. Reliability of a portable device for the detection of sleep bruxism. Clin Oral Investig. 2014;18(8):2037-43. doi: 10.1007/s00784-013-1168-z. [PubMed: 24374575].

22. Manfredini D, Ahlberg J, Castroflorio T, Poggio CE, Guarda-Nardini L, Lobbezoo F. Diagnostic accuracy of portable instrumental devices to measure sleep bruxism: a systematic literature review of polysomnographic studies. J Oral Rehabil. 2014;41(11):836-42. doi: 10.1111/joor.12207. [PubMed: 25040303].

23. Castroflorio T, Bargellini A, Rossini G, Cugliari G, Deregibus A, Manfredini D. Agreement between clinical and portable EMG/ECG diagnosis of sleep bruxism. J Oral Rehabil. 2015;42(10):759-64. doi: 10.1111/joor.12320. [PubMed: 26059761].

24. Castroflorio T, Deregibus A, Bargellini A, Debernardi C, Manfredini D. Detection of sleep bruxism: comparison between an electromyographic and electrocardiographic portable holter and polysomnography. J Oral Rehabil. 2014;41(3):163-9. doi: 10.1111/joor.12131. [PubMed: 24417585].

25. Farina D, Cescon C. Concentric-ring electrode systems for noninvasive detection of single motor unit activity. IEEE Trans Biomed Eng. 2001;48(11):1326-34. doi: 10.1109/10.959328. [PubMed: 11686631].

26. Koyano K, Tsukiyama Y, Ichiki R, Kuwata T. Assessment of bruxism in the clinic. J Oral Rehabil. 2008;35(7):495-508. doi: 10.1111/j.13652842.2008.01880.x. [PubMed: 18557916].

27. Lobbezoo F, Ahlberg J, Glaros AG, Kato T, Koyano K, Lavigne GJ, et al. Bruxism defined and graded: an international consensus. J Oral Rehabil. 2013;40(1):2-4. doi: 10.1111/joor.12011. [PubMed: 23121262].

28. Ravera S, Castroflorio T, Garino F, Daher S, Cugliari G, Deregibus A. Maxillary molar distalization with aligners in adult patients: a multicenter retrospective study. Prog Orthod. 2016;17:12. doi:10.1186/s40510016-0126-0. [PubMed: 27041551].

29. Rompre PH, Daigle-Landry D, Guitard F, Montplaisir JY, Lavigne GJ. Identification of a sleep bruxism subgroup with a higher risk of pain. J Dent Res. 2007;86(9):837-42. [PubMed:17720851].

30. Dube C, Rompre PH, Manzini C, Guitard F, de Grandmont P, Lavigne GJ. Quantitative polygraphic controlled study on efficacy and safety of oral splint devices in tooth-grinding subjects. J Dent Res. 2004;83(5):398-403. [PubMed:15111632]. 